\title{
A D-Band Rectangular Waveguide-to-Coplanar Waveguide Transition Using Metal Ridge
}

Dong, Yunfeng; Zhurbenko, Vitaliy; Hanberg, Peter Jesper; Johansen, Tom Keinicke

Published in:

Proceedings of 2019 IEEE MTT-S International Microwave Symposium

Publication date:

2019

Document Version

Peer reviewed version

Link back to DTU Orbit

Citation (APA):

Dong, Y., Zhurbenko, V., Hanberg, P. J., \& Johansen, T. K. (2019). A D-Band Rectangular Waveguide-toCoplanar Waveguide Transition Using Metal Ridge. In Proceedings of 2019 IEEE MTT-S International Microwave Symposium (pp. 1050-1053). IEEE.

\section{General rights}

Copyright and moral rights for the publications made accessible in the public portal are retained by the authors and/or other copyright owners and it is a condition of accessing publications that users recognise and abide by the legal requirements associated with these rights.

- Users may download and print one copy of any publication from the public portal for the purpose of private study or research.

- You may not further distribute the material or use it for any profit-making activity or commercial gain

- You may freely distribute the URL identifying the publication in the public portal

If you believe that this document breaches copyright please contact us providing details, and we will remove access to the work immediately and investigate your claim. 


\title{
A D-Band Rectangular Waveguide-to-Coplanar Waveguide Transition Using Metal Ridge
}

\author{
Yunfeng Dong ${ }^{\# 1}$, Vitaliy Zhurbenko ${ }^{\# 2}$, Peter Jesper Hanberg*3 ${ }^{* 3}$ Tom K. Johansen ${ }^{\# 4}$ \\ \#Electromagnetic Systems, Technical University of Denmark, Kgs. Lyngby, Denmark \\ *DTU Danchip, Technical University of Denmark, Kgs. Lyngby, Denmark \\ ${ }^{1}$ yundon, ${ }^{2} \mathrm{vz},{ }^{4}$ tkj@elektro.dtu.dk, ${ }^{3}$ jehan@danchip.dtu.dk
}

\begin{abstract}
This paper presents a rectangular waveguideto-coplanar waveguide (CPW) transition using metal ridge at D-band (110-170 GHz). The proposed transition is useful in particular for packaging circuits with large dimensions. A CPW with extended ground traces is designed on a quartz substrate and its performance is compared with a conventional CPW. Besides, an absorber layer is added underneath for restricting parasitic modes. As the critical part of the transition, the metal ridge is described in detail. The proposed rectangular waveguideto-CPW transition using metal ridge is designed, fabricated, and measured in a back-to-back configuration. The electric field distribution as well as the assembly of the proposed transition is illustrated. For the fabricated transition prototype in a back-toback configuration, the measured return loss remains better than $12.5 \mathrm{~dB}$ at D-band which corresponds to a bandwidth of $60 \mathrm{GHz}$. From $122.5 \mathrm{GHz}$ to $156.5 \mathrm{GHz}$, the measured insertion loss is less than $3 \mathrm{~dB}$ while it increases to $4 \mathrm{~dB}$ at the maximum. Thus, each fabricated transition contributes less than $2 \mathrm{~dB}$ insertion loss at D-band.
\end{abstract}

Keywords - integrated circuit packaging, millimeter wave devices, millimeter wave propagation, transmission lines.

\section{INTRODUCTION}

For system integration and packaging, rectangular waveguides serve as not only the transmission lines but also the interfaces for millimeter-wave and submillimeter-wave components due to its simple structure, low loss, and high powerhandling capability. However, the circuits as well as chips at such high frequencies rely on planar structures due to their fabrication processes. Under this circumstance, the transitions between rectangular waveguides and planar transmission lines have become a critical part determining the performances of the packaged components and systems which are under intensive study [1]-[10]. The challenging requirements for designing such transitions are wideband matching with low insertion loss and at the same time versatile for packaging the existing circuits and chips. According to [1], a rectangular waveguide-to-CPW transition between $220 \mathrm{GHz}$ and $325 \mathrm{GHz}$ was realized by tapering the width and height of the rectangular waveguide in several stages to match the characteristic impedance of the CPW. In [4], by inserting an E-plane probe into the rectangular waveguide through an aperture cut in the center of the broadwall located around a quarter wavelength away from the end, the designed transition was used for packaging amplifiers operating from $150 \mathrm{GHz}$ to $300 \mathrm{GHz}$. As is reported in [6], a wire bonding was implemented into a rectangular waveguide-to-CPW transition at D-band which achieves similar performances as the E-plane probe while the packaging approaches are more versatile. Besides,

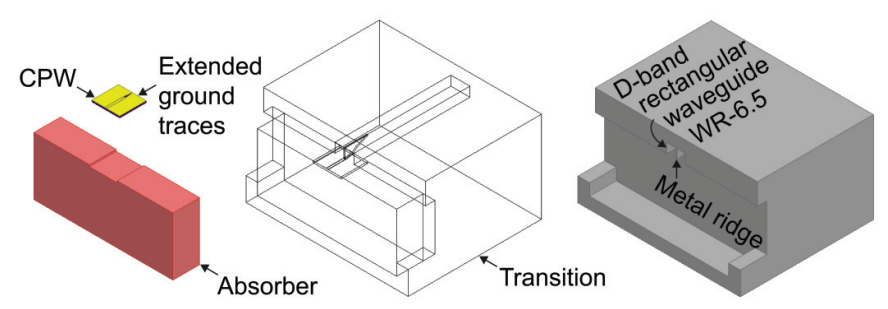

Fig. 1. Rectangular waveguide-to-CPW transition using metal ridge.

by placing a wideband planar antenna at the end of the rectangular waveguide in the direction of maximum radiation, the electromagnetic waves can be guided to the connected CPW [8], [9]. However, in this case the bandwidth of the transition is normally determined by the antenna. In general, the transition is realized on a separated substrate which can be connected to the chip using wire bondings, vias, or flip-chip approach. These chip-level connections introduce parasitic inductances and limit the bandwidth of the system especially at high frequencies. When the transition is integrated on-chip, the chip-level connections can be avoided while it may result in concave corners on the substrate since the transition is normally kept narrow for restricting parasitic modes in the closed cavity. Among other transition approaches, the metal ridge with a cosine shape introduced in [10] has been validated at K-band (18-26.5 GHz). Since the ridge and rectangular waveguide are applied to the $\mathrm{CPW}$ from the top, the transition also exhibits a potential for packaging circuits and chips with large dimensions.

Fig. 1 illustrates the basic structure of the proposed metal ridge transition between a WR-6.5 rectangular waveguide and a CPW with extended ground traces. The CPW through line patterned on the substrate represents the interface of millimeter-wave and submillimeter-wave circuits as well as chips. In addition, an absorber layer is placed under the substrate restricting parasitic modes and providing physical support since the CPW is aligned to the bottom broadwall of the rectangular waveguide. The metal ridge is realized inside the rectangular waveguide and connected to the signal trace of the CPW forming an in-line transition structure.

In this work, a D-band rectangular waveguide-to-CPW transition using metal ridge is designed and analyzed by using the full-wave electromagnetic simulator from Ansys. In order to validate the concept, a transition prototype is fabricated and measured in a back-to-back configuration. In section II, 


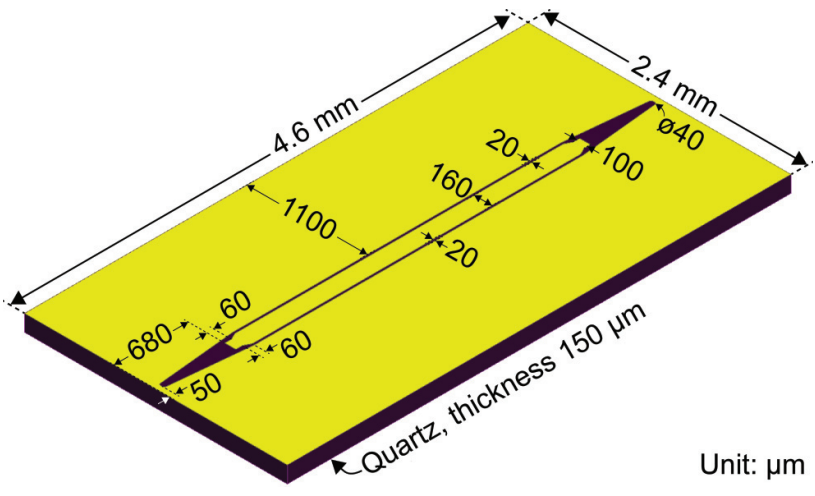

Fig. 2. Designed CPW with extended ground traces.

the designs of the $\mathrm{CPW}$ with extended ground traces and the metal ridge are introduced. The simulation results of the CPWs are presented and the electric field distribution of the proposed transition is illustrated. In section III, the fabrication, assembly, and experimental setup of the proposed transition is described and the measurement results are compared with the simulation results.

\section{Design Procedure of Rectangular WAVEGUIDE-TO-CPW TRANSITION}

\section{A. CPW with Extended Ground Traces}

Conventional CPW has an uniplanar structure where the signal trace is separated by two identical narrow gaps from the ground traces on both sides. The characteristic impedance is mainly determined by the ratio of $W /(W+2 G)$, in which $W$ and $G$ represent the widths of the signal trace and the gaps, respectively. A quasi-TEM mode is supported by the CPW and electric waves mainly exist in the gaps between the signal and ground traces. However, for CPWs based on thin substrates, part of the electric waves is coupled to the bottom ground plane which might result in mixed-mode propagations, radiations, and higher-order modes. In this case, either an absorber layer underneath or vias going through the substrate can be applied to restrict parasitic modes.

Fig. 2 shows the designed CPW as a part of the proposed rectangular waveguide-to-CPW transition. The thickness of the quartz $\left(\mathrm{SiO}_{2}\right)$ substrate is $150 \mu \mathrm{m}$. The material of the conductor layer is gold and it has a thickness of $400 \mathrm{~nm}$ by taking into account the skin depth at D-band. Compared with a conventional CPW, the ground traces are extended and merged at the end which works together with the metal ridge guiding electromagnetic waves to the rectangular waveguide. A $50 \Omega$ CPW through line in the middle and two extended ground traces are included in the design. The length and width of the substrate are $4.6 \mathrm{~mm}$ and $2.4 \mathrm{~mm}$, respectively, which are comparable dimensions as large circuits at D-band. The CPW through line in the middle serves as the substitution of the circuits on the substrate. Besides, being similar to chip interfaces, the width of the signal trace is tapered to $100 \mu \mathrm{m}$ at the end in order to fit with on-wafer measurements.

The solid lines in Fig. 3 represent the simulation results of the CPW with extended ground traces. In comparison, the

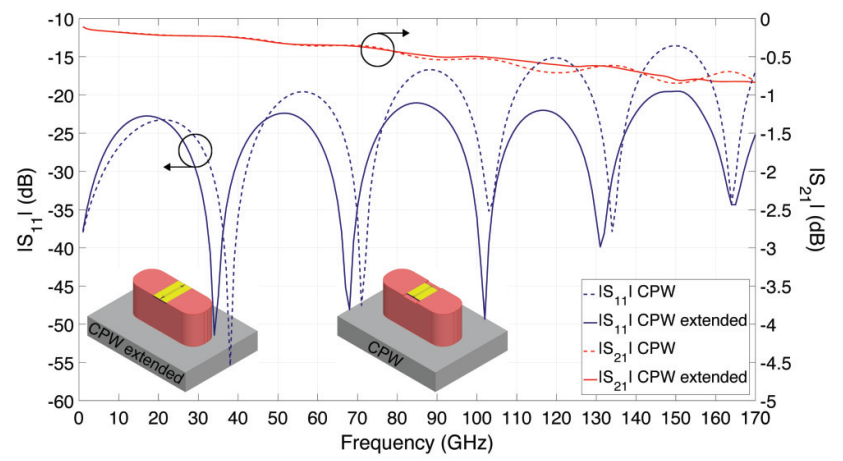

Fig. 3. Simulation results of the CPWs based on quartz substrate.

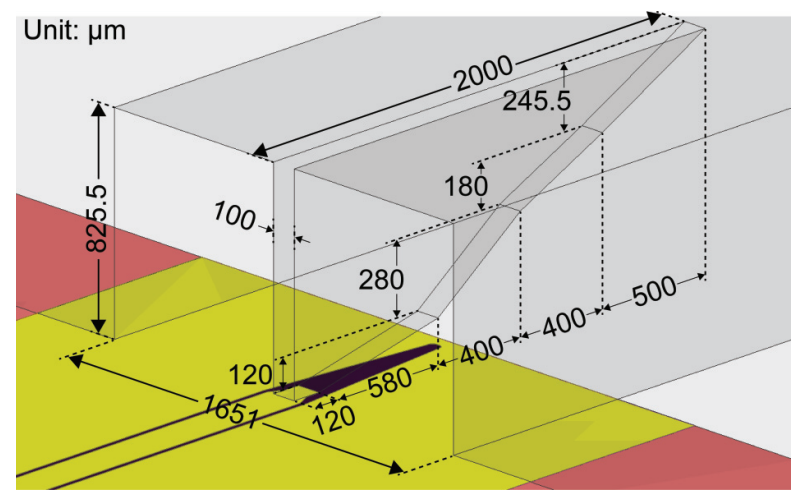

Fig. 4. Designed metal ridge transition.

dashed lines show the simulation results of the CPW through line in the middle. The simulation structures for both situations are presented. Lumped ports with vertical perfect electric conductor (PEC) bridges are used as the excitation scheme in the simulations. The material of the absorber layer is Rohacell (31-HF) whose dielectric constant $\left(\epsilon_{r}\right)$ is around 1. Similar performances are achieved up to $170 \mathrm{GHz}$ and the return loss is better than $13 \mathrm{~dB}$ with an associated insertion loss of $1 \mathrm{~dB}$. Since the extended ground traces do not affect the performance of the circuits in the middle, it becomes possible to measure the circuits at the beginning and package it afterwards without extra structures on the substrate or chip-level connections.

\section{B. Metal Ridge Transition}

Fig. 4 demonstrates the metal ridge transition where the ridge and the sidewalls are connected to the signal and ground traces of the CPW, respectively. Since part of the bottom broadwall of the rectangular waveguide is cut and connected to the extended ground traces of the CPW, the absorber layer underneath also provides physical support to the substrate. The ridge has a length of $2 \mathrm{~mm}$ including 5 stages which starts gradually on the top broadwall of the rectangular waveguide and connects to the tapered signal trace at the end. Besides, the thickness of the metal ridge is designed to be $100 \mu \mathrm{m}$ in order to fit the width of the tapered signal trace while accurate alignment is required in this case.

The proposed metal ridge transition is simulated in a back-to-back configuration which consists of two WR-6.5 rectangular waveguides, two identical transitions and a CPW 


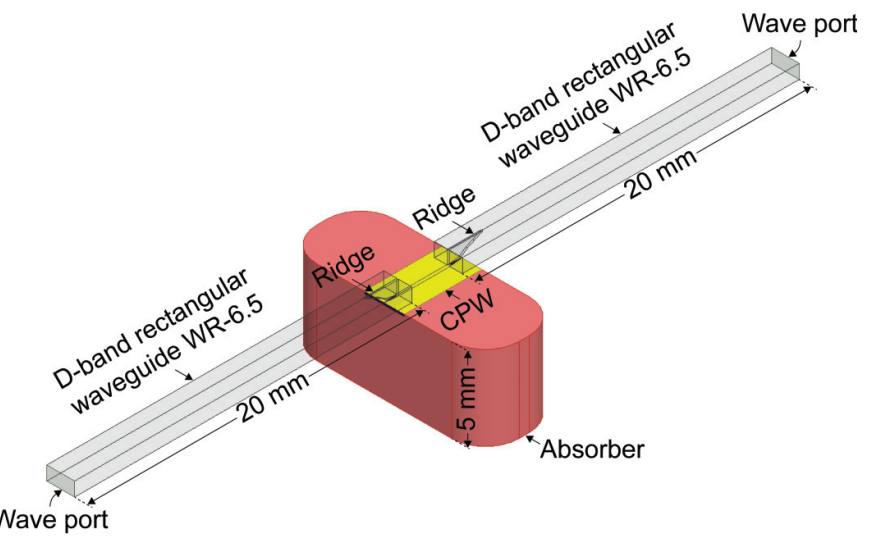

Wave port

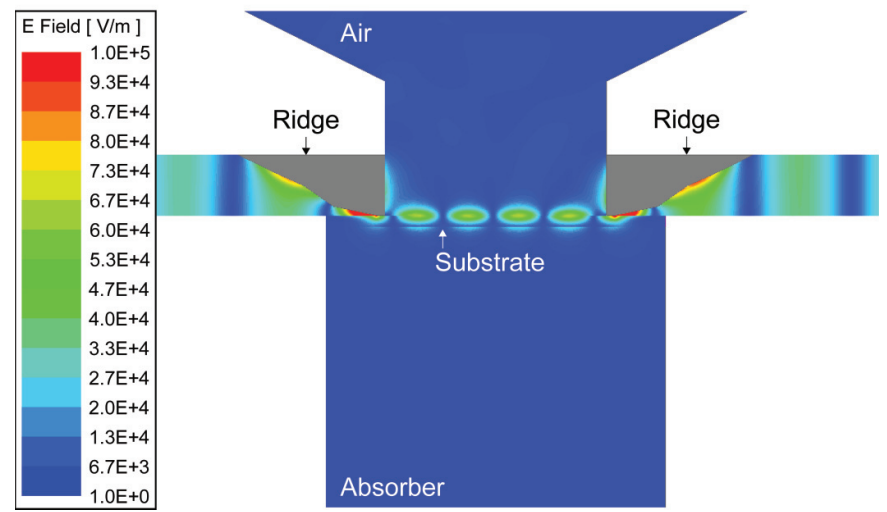

Fig. 5. Simulation structure of the proposed ridge transition in a back-to-back configuration and the magnitude of electric field distribution at $140 \mathrm{GHz}$.

in the middle. The simulation structure is shown in Fig. 5 and wave ports are used as the excitation scheme. The length of the rectangular waveguide is $20 \mathrm{~mm}$ and the absorber layer has a thickness of $5 \mathrm{~mm}$. Fig. 5 also illustrates the magnitude of electric field distribution at the cross-section along the E-plane in the middle of the rectangular waveguide. At the beginning of the transition, the electric waves in the rectangular waveguide are coupled to the metal ridge gradually and change direction from vertical to horizontal due to the extended ground traces. When the metal ridge approaches the signal trace of the $\mathrm{CPW}$, the electric waves concentrate between the metal ridge and the ground traces of the CPW which convert to a $\mathrm{CPW}$ mode and propagate on the substrate at the end of the transition. Besides, the refraction of electric waves can be clearly observed on the edge between the quartz substrate and the absorber layer. The leakage of electric waves through the aperture at the end of the rectangular waveguide can be neglected.

\section{FABRICATION AND EXPERIMENTAL RESULTS}

In order to validate the concept, the proposed rectangular waveguide-to-CPW transition using metal ridge shown in Fig. 4 was fabricated in a back-to-back configuration. Fig. 6 demonstrates the assembly structure, fabricated CPW, and packaged transition prototype. The CPW with extended ground traces was fabricated in the cleanroom where the quartz substrate was cleaned by sputtering at the beginning. After that a $30 \mathrm{~nm}$ titanium layer for increasing the adhesion

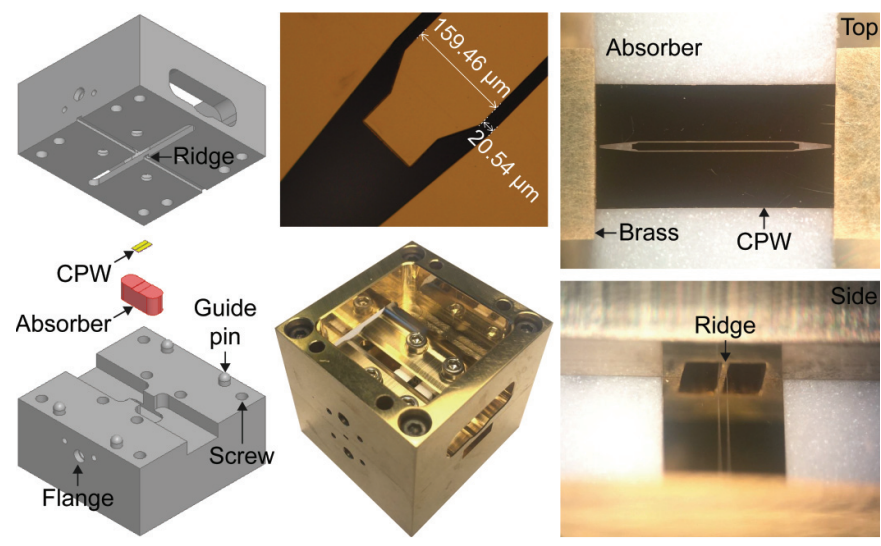

Fig. 6. Assembly and fabrication of the rectangular waveguide-to-CPW transition using metal ridge.

and a gold layer with a thickness of $400 \mathrm{~nm}$ were deposited on the quartz substrate. The CPW was patterned by following the process of photolithography in which a maskless aligner was used for ultraviolet (UV) exposure and potassium iodide (KI) was used for wet chemical etching. The layout was repeated on the substrate which were cut into samples by using a dicing saw. In order to overcome the stress, the quartz substrate was attached to a silicon wafer during the dicing process. The dimensions of the fabricated CPW were verified visually by measuring under a microscope.

For easier fabrication and substrate alignment, the packaging structure was divided into two parts and each part was fabricated using an brass block by a milling process which uses a spinner with a radius of $250 \mu \mathrm{m}$. The WR6.5 rectangular waveguides and metal ridges were realized on the top part while the bottom part contains a recess with a depth of $1 \mathrm{~mm}$ for aligning the absorber layer. Besides, two standard rectangular waveguide flanges were also fabricated at the input and output of the packaging structure. For assembly, the CPW was placed inside the slot with a depth of $150 \mu \mathrm{m}$ on top of the absorber layer. Sliver epoxy was used not only for filling the gaps between the ground traces and the rectangular waveguide but also for connecting the metal ridge to the signal trace. The top and bottom parts were combined by using guide pins as well as screws which guarantee an accurate alignment and tight connections. The packaged transition prototype was inspected visually through the opening on the top. It can be observed that the metal ridge was aligned accurately to the signal trace of the CPW.

Fig. 7 illustrates the experimental setup for measuring the proposed rectangular waveguide-to-CPW transition in a backto-back configuration at D-band. The measurement system consists of an Anritsu ME7808B vector network analyzer (VNA) and two VDI WR-6.5 waveguide extenders which can measure two-port scattering parameters of the device under test (DUT) from $110 \mathrm{GHz}$ to $170 \mathrm{GHz}$. For improving the accuracy, through-reflect-line (TRL) calibration was applied to the measurement by using a waveguide calibration kit. The measured scattering parameters were calibrated to the input and output of the fabricated transition prototype. 


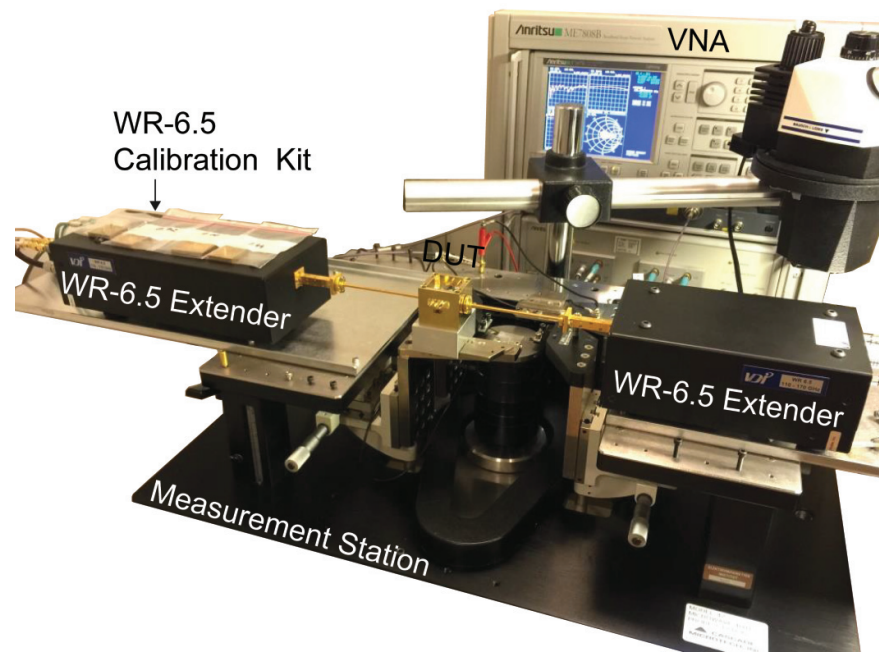

Fig. 7. Experimental setup showing the device under test, vector network analyzer, WR-6.5 waveguide extenders, and calibration kit.

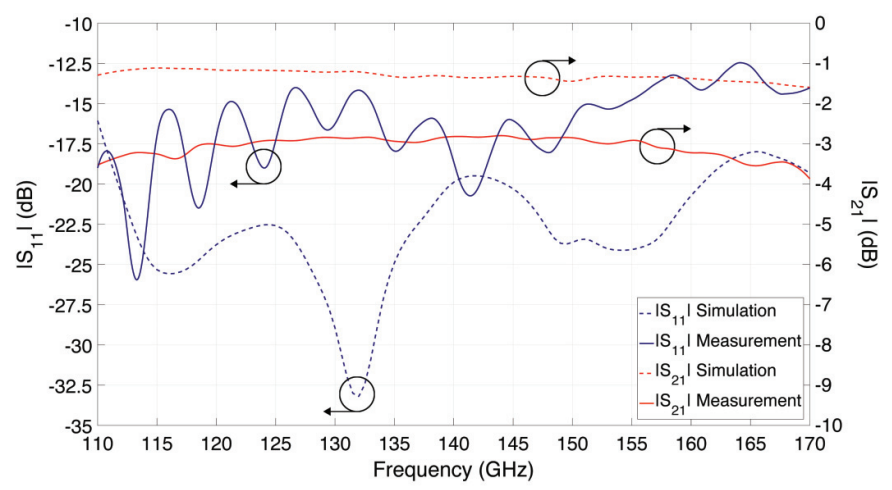

Fig. 8. Simulation and measurement results of the proposed the rectangular waveguide-to-CPW transition using metal ridge.

The measurement results of the proposed rectangular waveguide-to-CPW transition in a back-to-back configuration are shown by the solid lines in Fig. 8. In comparison, the dashed lines represent the simulation results. The measured return loss remains better than $12.5 \mathrm{~dB}$ from $110 \mathrm{GHz}$ to $170 \mathrm{GHz}$ which corresponds to a bandwidth of $60 \mathrm{GHz}$ at D-band. For the measured insertion loss, it is less than $3 \mathrm{~dB}$ from $122.5 \mathrm{GHz}$ to $156.5 \mathrm{GHz}$ and still less than $4 \mathrm{~dB}$ at the maximum. Thus, each fabricated rectangular waveguideto-CPW transition using metal ridge contributes less than $2 \mathrm{~dB}$ insertion loss at D-band. Compared with the simulation results, a reasonable agreement is achieved while the differences are mainly caused by the alignment and packaging tolerances especially the gaps between the ground traces of the CPW and the rectangular waveguide. In addition, the connection between the signal trace and the metal ridge is a critical part which affects the performances of the transition in particular the insertion loss.

\section{CONCLUSION}

The design of rectangular waveguide-to-CPW transition using metal ridge at D-band has been presented. As a part of the proposed transition, a CPW based on quartz substrate with extended ground traces has been introduced and compared with a conventional CPW. In both cases, an absorber layer was implemented for restricting parasitic modes and providing physical support. The metal ridge has been described in detail and the electric field distribution of the proposed transition was illustrated. The transition prototype was fabricated and measured in a back-to-back configuration which validates the concept of using metal ridge at D-band as a transition between a WR-6.5 rectangular waveguide and a CPW. The measured return loss remains better than $12.5 \mathrm{~dB}$ at $\mathrm{D}$-band while each fabricated transition introduces less than $2 \mathrm{~dB}$ insertion loss. Since the extended ground traces on both sides of the substrate do not affect the performance of the CPW through line in the middle, it can also be applied to other circuits as well as chips. In this way, the proposed transition makes it possible to test the circuit on-wafer and then package it into a waveguide component without adding extra structures on the substrate or any chip-level connections. It is useful especially for packaging circuits with large dimensions.

\section{ACKNOWLEDGMENT}

The authors would like to thank M. Nielsen, J. Lauridsen, and J. N. Mortensen at the Technical University of Denmark for machining the packaging structures.

\section{REFERENCES}

[1] M. Moallem, J. East, and K. Sarabandi. "A broadband, micromachined rectangular waveguide to cavity-backed coplanar waveguide transition using impedance-taper technique," IEEE Trans. Compon. Packag. Manuf. Technol., vol. 4, pp. 49-55, Jan. 2014.

[2] M. Vahidpour and K. Sarabandi. "2.5D micromachined $240 \mathrm{GHz}$ cavitybacked coplanar waveguide to rectangular waveguide transition," IEEE Trans. THz Sci. Technol., vol. 2, pp. 315-322, May. 2012.

[3] S. Jameson and E. Socher, "A wide-band CMOS to waveguide transition at mm-wave frequencies with wire-bonds," IEEE Trans. Compon. Packag. Manuf. Technol., vol. 63, pp. 2741-2750, Sept. 2015.

[4] K. Eriksson, P. J. Sobis, S. E. Gunnarsson, J. Hanning, and H. Zirath, "InP DHBT amplifier modules operating between 150-300 GHz using membrane technology," IEEE Trans. Compon. Packag. Manuf. Technol., vol. 63, pp. 433-440, Feb. 2015.

[5] V. Hurm, R. Weber, A. Tessmann, H. Massler, A. Leuther, M. Kuri, M. Riessle, H. P. Stulz, M. Zink, M. Schlechtweg, O. Ambacher, and T. Närhi, "A $243 \mathrm{GHz}$ LNA module based on mHEMT MMICs with integrated waveguide transitions," IEEE Microw. Compon. Lett., vol. 23, pp. 486-488, Sept. 2013.

[6] Y. Dong, V. Zhurbenko, P. J. Hanberg, and T. K. Johansen, "A Dband rectangular waveguide-to-coplanar waveguide transition using wire bonding probe," J. Infrared Millim. Terahertz Waves, pp. 1-17, Nov. 2018.

[7] Y. Dong, T. K. Johansen, V. Zhurbenko, and P. J. Hanberg, "Rectangular waveguide-to-coplanar waveguide transitions at U-band using E-plane probe and wire bonding," in Proc. 46th Eur. Microw. Conf. (EuMC), pp. 5-8, Oct. 2016.

[8] Y. Dong, T. K. Johansen, V. Zhurbenko, and P. J. Hanberg, "A rectangular waveguide-to-coplanar waveguide transition at D-band using wideband patch antenna," in Proc. 48th Eur. Microw. Conf. (EuMC), pp. $1-4$, Sept. 2018.

[9] T. Tajima, H. J. Song, H. Matsuzaki, and M. Yaita, "LTCC-integrated $\mathrm{H}$-plane bends for $\mathrm{THz}$ antenna-in-package solution," IEEE Microw. Compon. Lett., vol. 27, pp. 440-442, May. 2017.

[10] G. E. Ponchak and R. N. Simons, "A new rectangular waveguide to coplanar waveguide transition," in Proc. IEEE MTT-S Int. Microwave Symp. Dig., pp. 491-492, May. 1990. 